Research Paper

\title{
Extracellular Matrix of Mechanically Stretched Cardiac Fibroblasts Improves Viability and Metabolic Activity of Ventricular Cells
}

\author{
Yong Guo ${ }^{1}$, Qiang-cheng Zeng ${ }^{2}{ }^{凶}$, Chun-qiu Zhang ${ }^{3}$, Xi-zheng Zhang ${ }^{1 凶}$, Rui-xin Li ${ }^{2}$, Ji-min Wu ${ }^{1}$, Jing \\ Guan ${ }^{1}$, Lu Liu ${ }^{4}$, Xin-chang Zhang ${ }^{1}$, Jian-yu Li1 ${ }^{1}$, Zong-ming Wan ${ }^{1}$ \\ 1. Lab of Biomaterial, Tianjin Institute of Medical Equipment, Academy of Military Medical Science, Tianjin, China; \\ 2. Shandong Provincial Key Laboratory of Functional Macromolecular Biophysics, Institute of Biophysics, Dezhou University, Dezhou, China; \\ 3. School of Mechanical Engineering, Tianjin University of Technology, Tianjin, China; \\ 4. Chemistry department, Logistics College of Chinese Peoples Armed Police Forces. Tianjin, 300162, China. \\ $\triangle$ Corresponding authors: Qiang-cheng Zeng Ph.D., Xi-zheng Zhang Ph.D., qiangchengzeng@126.com, z56717@sohu.com, Institute of Biophysics, Dezhou \\ University, No. 566 Daxuexi Road, Decheng District, Dezhou City, 253021, China. Tel: +8613805340914
}

( ) Ivyspring International Publisher. This is an open-access article distributed under the terms of the Creative Commons License (http://creativecommons.org/ licenses/by-nc-nd/3.0/). Reproduction is permitted for personal, noncommercial use, provided that the article is in whole, unmodified, and properly cited.

Received: 2013.05.29; Accepted: 2013.08.26; Published: 2013.II.05

\begin{abstract}
Background: In heart, the extracellular matrix (ECM), produced by cardiac fibroblasts, is a potent regulator of heart's function and growth, and provides a supportive scaffold for heart cells in vitro and in vivo. Cardiac fibroblasts are subjected to mechanical loading all the time in vivo. Therefore, the influences of mechanical loading on formation and bioactivity of cardiac fibroblasts' ECM should be investigated.

Methods: Rat cardiac fibroblasts were cultured on silicone elastic membranes and stimulated with mechanical cyclic stretch. After removing the cells, the ECMs coated on the membranes were prepared, some ECMs were treated with heparinase II (GAG-lyase), then the collagen, glycosaminoglycan (GAG) and ECM proteins were assayed. Isolated neonatal rat ventricular cells were seeded on ECM-coated membranes, the viability and lactate dehydrogenase (LDH) activity of the cells after I-7 days of culture was assayed. In addition, the ATPase activity and related protein level, glucose consumption ratio and lactic acid production ratio of the ventricular cells were analyzed by spectrophotometric methods and Western blot.

Results: The cyclic stretch increased collagen and GAG levels of the ECMs, and elevated protein levels of collagen I and fibronectin. Compared with the ECMs produced by unstretched cardiac fibroblasts, the ECMs of mechanically stretched fibroblasts improved viability and LDH activity, elevated the $\mathrm{Na}^{+} / \mathrm{K}^{+}$-ATPase activity, sarco(endo)plasmic reticulum Ca ${ }^{2+}$-ATPase (SERCA) activity and SERCA 2a protein level, glucose consumption ratio and lactic acid production ratio of ventricular cells seeded on them. The treatment with heparinase II reduced GAG levels of these ECMs, and lowered these metabolism-related indices of ventricular cells cultured on the ECMs.

Conclusions: Mechanical stretch promotes ECM formation of cardiac fibroblasts in vitro, the ECM of mechanically stretched cardiac fibroblasts improves metabolic activity of ventricular cells cultured in vitro, and the GAG of the ECMs is involved in regulating metabolic activity of ventricular cells.
\end{abstract}

Key words: mechanical stretch, cardiac fibroblast, extracellular matrix, glycosaminoglycan, metabolism.

\section{Introduction}

ECM is secreted by resident cells of tissue, it provides mechanical support for cells' growth and affects cell adhesion, proliferation, differentiation, morphology and gene expression (1). ECM is a potent regulator of cell function and differentiation, and provides a supportive scaffold and microenvironment 
for mammalian cells in vitro and in vivo $(2,3)$. Therefore, from the view of biomimetics, ECM is the most suitable scaffold for cell/tissue culture.

The ECM produced by cardiac fibroblasts is the major component of cardiac tissue $(4,5)$. Cardiac fibroblasts are constantly subjected to mechanical stretch in vivo, and responsive to mechanical stimuli, they synthesize and degrade the ECM which provides a 3D network for cardiomyocytes and other cells of the heart to ensure proper cardiac form and function (5). Therefore, the investigation of mechanical stimuli influencing formation and bioactivity of ECM, especially produced in vitro, presents a particularly promising line of research in the field of cardiac cell/tissue engineering.

Cardiac fibroblast is mechano-responsive, it can transform mechanical stimuli into biochemical signals. Mechanical stretch increased mRNA levels of collagen type III and collagen type I of cardiac fibroblasts $(6,7)$, also increased mRNA levels of matrix metalloproteinase- 2 and tissue inhibitor of matrix metalloproteinase-2 (8). In these studies, the mRNAs of collagen and other ECM-related genes did not directly represent actual protein levels of ECM. In fact, the effect of mechanical stimuli on ECM formation in vitro is not fully understood.

In recent years, there has been considerable investigation of the bioactivity of cardiac fibroblast-derived ECM (CF-ECM) which was coated on cell culture dishes in vitro. The CF-ECM was shown to support early maturation of embryonic stem cell-derived cardiomyocytes, in terms of chronotropic characteristics and subcellular structural development (9). In addition, CF-ECM increased proliferation, improved tolerance to oxidative stress and adhesion potential of bone marrow-derived stem cells (10). Furthermore, the proliferative activity of MC3T3-E1 cells cultured on CF-ECM was higher than that on osteoblast-derived ECM (11). These studies are likely to contribute to ECM-modified scaffold for cell/tissue engineering. However, the effect of mechanical strain on bioactivity of CF-ECM remains unexplored.

In this study, we stimulated rat cardiac fibroblasts cultured on silicone elastic membranes with mechanical cyclic stretch, prepared the ECM-coated membranes, then detected collagen, fibronectin and GAG in the ECMs, assessed metabolic activity of neonatal rat ventricular cells which were seeded on the ECM-coated membranes. The control groups consisted of membranes coated with ECM produced by unstretched cardiac fibroblasts or uncoated membranes. Thus, the in vitro effects of mechanical stretch on formation and bioactivity of CF-ECM were investigated.

\section{Materials and methods}

\section{Preparation of cardiac fibroblast-derived ECM-coated cell culture dishes}

Cardiac fibroblasts from the ventricles of 3-day old Wistar rats [isolated and cultured by our lab (12)] were cultured on silicone elastic membranes (Specialty Manufacturing, Saginaw, MI, USA) in complete alpha minimal essential medium (a-MEM; Invitrogen, Carlsbad, CA, USA) supplemented with $10 \%$ fetal calf serum and $1 \%$ penicillin-streptomycin. At confluence, the cells on the membranes were cultured in complete a-MEM supplied with $280 \mu \mathrm{mol} / \mathrm{L}$ ascorbic acid, subjected to cyclic stretch of $4 \%$ or $8 \%$ at $1.0 \mathrm{~Hz}, 1 \mathrm{~h}$ per/day for indicated times. The mechanical stretch was generated by a custom-made cell stretch apparatus described previously (13). The device was driven by a stepping motor (controlled by a single chip microcomputer), it uniformly stretched silicone elastic membranes, resulting in homogeneous equibiaxial strains applied to the cultured cells on the membranes.

The cells were eliminated according to our established method $(12,14)$. After washing with PBS, the cells were removed by incubation for $3 \mathrm{~min}$ with PBS containing $0.5 \%$ Triton X-100 and $10 \mathrm{mM} \mathrm{NH}_{4} \mathrm{OH}$ at room temperature then rinsed three times with PBS. The ECMs attached to the elastic membranes were treated with 100 units/ml DNase (Sigma-Aldrich, St. Louis, MO, USA) for $1 \mathrm{~h}$ and the resulting ECMs were washed with PBS, observed by inverted microscopy (Figure 1), then stored at $4^{\circ} \mathrm{C}$ for further use. Unstretched cultures (control group) were maintained under identical culture conditions without mechanical loading.

\section{Assay of ECM}

A. Measure hydroxyproline content. The ECMs which coated on the menbranes were hydrolyzed and the hydroxyproline content were detected with the Chloramine-T Hydroxyproline Assay Kit containing hydrolysis buffer (Nanjing Jiancheng Biotechnology Institute Co., Ltd., Nanjing, China.) according to the manufacturer's protocol.

B. GAG analysis. According to the manufacturer's protocol, the ECMs were treated with lysis buffer and GAG levels were measured with Dimethyl-methylene Blue GAG Assay Kit (Xianmen Maiwei Biotechnology Co., Ltd., Xiamen, China.). The absorbance or optical density (OD) at $660 \mathrm{~nm}$ was regarded as relative level of GAG in the ECMs. Results were expressed as relative to control group (the ECM of unstretched cells seeded on membranes). 


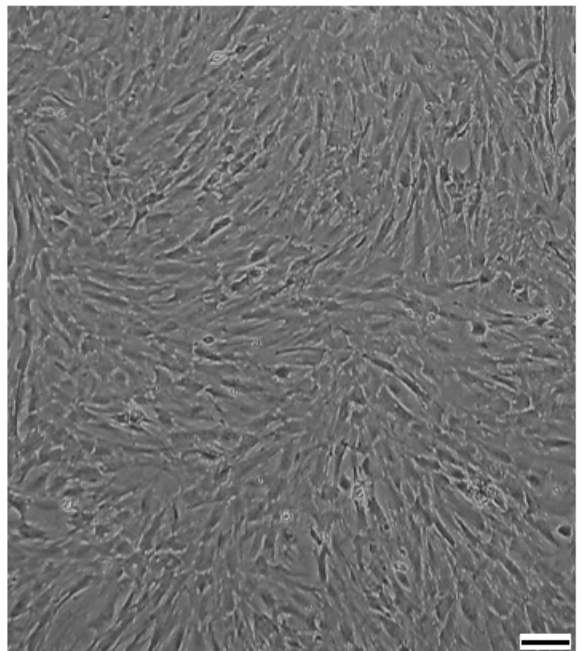

cell were cultured

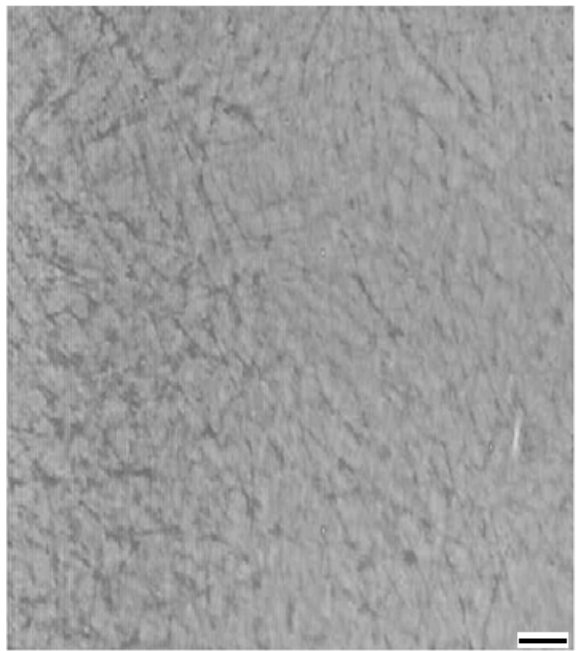

cell were removed bar: $100 \mathbf{u m}$

Fig I. Preparation of cardiac fibroblast ECM which was coated on dishes.

C. Van Gieson Staining. Some of the ECMs on the menbranes were fixed with 3\% paraformaldehyde. According to the supplier's protocol, the ECMs were stained with Van Gieson Histochemical Staining Kit (Nanjing Jiancheng Biotechnology Co. Ltd, China, containing hematoxylin solution) to dye the collagen fibers red, and nuclei blue.

\section{Isolating of Neonatal ventricular cells}

The ventricular cells were isolated according to our previous method (15). The ventricles of rat hearts were minced, rinsed with PBS consisting of $1 \%$ penicillin streptomycin (Invitrogen), and treated with four times of 6-minute digestion in $0.25 \%$ trypsin solution (Invitrogen) and one time 20-minute digestion in $0.1 \%$ collagenase type I (Invitrogen) solution at $37^{\circ} \mathrm{C}$ on a shaker $(140 \mathrm{r} / \mathrm{min})$. All supernatants except the first digestate were centrifuged at $1,000 \mathrm{r} / \mathrm{min}$ for 5 minutes, and the red cells were removed by Red blood Cell Lysis Buffer (Beijing Apply Gene Technology Co.. Ltd., Beijing, China). The resulting pellet was resuspended in high-glucose Dulbecco's modified minimal essential medium (Invitrogen) containing $10 \%$ fetal bovine serum and $1 \%$ penicillin-streptomycin.

\section{MTT assay}

Ventricular cells at a density $2.5 \times 10^{5} / \mathrm{cm}^{2}$ were seeded on ECM-coated membranes, the cells were treated with $0.1 \mathrm{mmol} / \mathrm{L}$ 5-Bromo-2'-deoxyuridine (BrdU, Sigma, inhibit proliferation of cells). One, 3 or 5 days later, MTT (3-(4, 5-dimethylthiazol-2-yl)-2, 5-diphenyltetrazolium bromide) solution (Promega, $1 \mathrm{mg} / \mathrm{mL}$ ) was used to assay living cells according to the manufacturer's protocol. In this assay, MTT is reduced to formazan by intracellular NAD (P)
H-oxidoreductase. The formazan product can be detected using an enzyme-linked immunosorbent assay reader at $490 \mathrm{~nm}$. The absorbance or OD at $490 \mathrm{~nm}$ was regarded as an index of the relative number of viable adherent cells and relative activity of cell growth (16).

\section{Assay of lactate dehydrogenase (LDH)}

After the ventricular cells were incubated with LDH leakage buffer and centrifuged for 5 minute, the activity of $\mathrm{LDH}$ in leakage buffer was measured spectrophotometrically at $490 \mathrm{~nm}$ with LDH Assay Kit (Nanjing Jiancheng Biotechnology Institute Co., Ltd.), according to the manufacturer's protocol. In this assay, LDH catalyzed the reduction of NAD to $\mathrm{NADH}$, then INT (2-p-iodophenyl-3-nitrophenyl tetrazolium chloride) was reduced to red formazan by diaphorase. The absorbance or OD at $490 \mathrm{~nm}$ was regarded as relative content of $\mathrm{LDH}$ leakage. Results were expressed as relative to control group (the cells on membranes without ECM coating).

\section{ATPase activity assays}

After trypsinization of $0.2 \%$ trypsin and centrifugation, the cells were lysed by brief sonication on ice in a lysis buffer $(10 \mathrm{mmol} / \mathrm{L}$ HEPES, $250 \mathrm{mmol} / \mathrm{L}$ sucrose, $5 \mathrm{mmol} / \mathrm{L}$ Tris-HCl, $1 \mathrm{mmol} / \mathrm{L}$ EDTA, $0.01 \%$ TritonX-100, pH 7.5). According to the protocol provided by the manufacturer, the protein concentration of the cell lysates were measured with the BCA protein assay kit (Beijing Applygen Genetechnology Inc, Beijing, China). The $\mathrm{Na}^{+} / \mathrm{K}^{+}$-ATPase and SERCA $\left(\mathrm{Ca}^{2+}\right.$-ATPase) activity of the cell lysates was assayed respectively with ATPase activity assay kits (Nanjing Jiancheng Biotechnology Co. Ltd, China) according to the supplier's protocol. The ATPase activity was 
showed as the inorganic phosphorous $(\mathrm{Pi})$ production from the decomposition per $\mathrm{mg}$ protein per hour ( $\mu \mathrm{molPi} / \mathrm{mg} \cdot \mathrm{h}$ ).

\section{Measure of glucose consumption ratio and lactic acid production ratio}

After one days of incubation with renewed culture medium, the glucose and lactic acid contents in the culture medium were measured with Glucose assay kit and Lactic acid assay kit (Nanjing Jiancheng Biotechnology Co. Ltd, Nanjing, China), according to the supplier's protocols. The glucose consumption ratio was the amount of glucose consumed (decrease of glucose content) by $10^{6}$ cells in one day ( $\mu \mathrm{mol} / \mathrm{million}$ cells/day). The lactic acid production ratio was the amount of lactic acid produced (increase of lactic acid) by $10^{6}$ cells in one day $(\mu \mathrm{mol} / \mathrm{million}$ cells/day).

\section{Western blot analysis}

Total cell lysates were prepared in RIPA lysis buffer containing protease inhibitors. Protein concentration was determined according to Brandford's method. Each aliquot of $10 \mu \mathrm{g}$ protein was subjected to electrophoresis on a polyacrylamide gel containing 0.1\% SDS (SDS-PAGE) and electro-transferred onto polyvinylidene difluoride membranes (Millipore, Bedford, MA, USA). After blocking with 5\% skim milk, the membranes were incubated overnight respectively with the mouse anti-collagen I [Abcam(HK) Ltd.], rabbit anti-fibronectin (Wuhan Boster Biotechnology Co.Ltd., Wuhan, China) and rabbit anti-SERCA2a (Wuhan Boster) then incubated with horseradish peroxidase-conjugated secondary antibody. The immunoreactive bands were visualized using an enhanced chemiluminescence detection kit (Wuhan Boster). Optical density of the protein bands was determined with Gel Doc 2000 (Bio-Rad, CA, USA). The expression of glyceraldehyde3-phosphatedehydrogenase (GAPDH) of the cells was used as a loading control, data were normalized against those of corresponding GAPDH.

\section{Statistical analysis}

The data are presented as mean \pm standard deviation, and analyzed by SPSS 13.0 software (Chicago, IL, USA) using one-way ANOVA. A value of $\mathrm{P}<0.05$ was considered statistically significant.

\section{Results}

Mechanical stretch increased collagen, GAG content in CF-ECM and elevated fibronectin and collagen I protein levels of cardiac fibroblast

After subjecting cardiac fibroblasts to a mechan- ical stretch of $4 \%$ or $8 \%$ at $1 \mathrm{~Hz}, 1 \mathrm{~h} /$ day, the hydroxyproline and GAG content of the ECM which were produced by the mechanically stretched cells and attached to membranes, were both increased, compared with those of ECM produced by unstretched cells (control group) (Fig. 2 A, B). In addition, along with the duration of culturing, the hydroxyproline were both significantly increased for 9 days (Fig. 2A). Resulting hydroxyproline measurements were converted to collagen contents following a 1: 10 (hydroxyproline: collagen) ratio (17). So the change of hydroxyproline equated to the change of collagen content.

The results of Van Gieson straining confirmed that mechanical stretch increased collagen level of ECM (Fig. 2C). In addition, Western blot assay indicated that mechanical stretch elevated fibronectin protein and collagen I protein levels of cardiac fibroblast (Fig. 2D).

The ECM, produced by mechanically stretched cells, enhanced viability and LDH activity of ventricular cells seeded on it

The MTT assay was used to evaluate the viability of the cultured cardiac cells (17). After the ventricular cells were seeded on the membranes coated with the ECM of the cells which were stimulated with mechanical stretch for 7 days, the OD values of MTT assay were all higher than the cells re-seeded on the ECM produced by the unstetched cells (unstretch group) or the group without ECM coating (Fig. 3A). Additionally, the LDH activity were all higher than the ECM of unstretched cells or the group without ECM (Fig. 3B). LDH is a soluble enzyme and localized in the cytoplasm, it is used as a quantitative marker enzyme for the intact cell, its activity providing information on viability or cellular glycolytic capacity (18). Therefore, these results indicated that the ECM of the mechanically stretched cells enhanced viability or glycolytic capacity.

The ECM of mechanically stretched cells enhanced ATPase activity, glucose consumption and lactic acid production ratios, and elevated SERCA 2 a level

After culturing for 1, 3 and 5 days on the ECM of mechanically stretched cardiac fibroblasts, the ATPase $\left(\mathrm{Na}^{+} / \mathrm{K}^{+}\right.$-ATPase and SERCA, important index of cardiac cell function and metabolism) activities were all higher than that of unstretched cells (Fig. 4A). Glucose consumption ratio and lactic acid production ratio (important indices of cell metabolism) were also higher (Fig. 4B). After 3 days of culturing, the protein levels of SERCA2a was increased (Fig. 4C). These results confirmed that the ECM of the mechanically 
stretched cells enhanced metabolic activity.

The ECM which was treated with heparinase, lowered ATPase activity, LDH activity, glucose consumption and lactic acid production ratios of ventricular cells

After the ECMs were treated with heparinase II, the GAG levels of the ECM were reduced (Fig. 2B). The ATPase activity, LDH activity, glucose consumption and lactic acid production ratios of ventricular cells seeded on them were all decreased evidently
(Fig. 5A-C). Moreover, the protein level of SERCA2a was reduced (Fig. 5D). Heparinase II is a broad substrate-specificity GAG-lyase, it can decompose GAG. Heparinase II treatment reduced GAG level of ECMs (Fig. 2B). Notablely, after heparinase II treatment, the metabolism-related indices of the ventricular cells on ECM of stretched cells were nearly as same as the indices of the ventricular cells on ECM of unstretched cells (Fig. 5A-D).
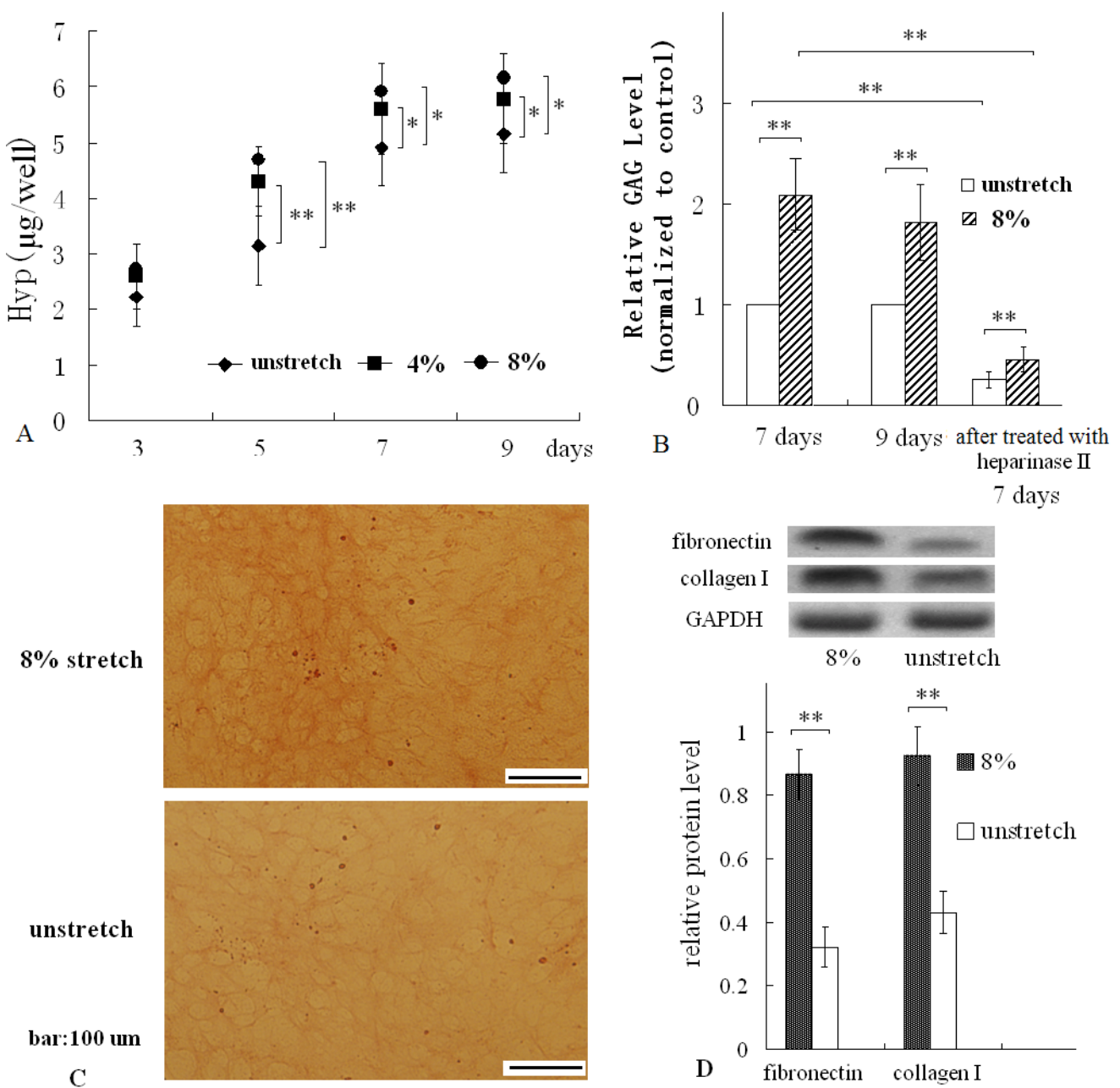

Fig 2. Hydroxyproline (Hyp) and GAG assay, Van Gieson straining of the ECM coated on dishes, and Western blot of fibronectin and collagen I. (A) The mechanical stretch (4\% or $8 \%$ ) increased the contents of hydroxyproline at the indicated times; (B) the mechanical stimulus for 7 days or 9 days enhanced GAG levels of the ECM, and treatment with hyaluronidase II reduced GAG levels remarkably; (C) Van Gieson straining showed more collagen fibers (which were stained red) in stretched group; (D) Western blot result indicated that mechanical stretch elevated protein levels of fibronectin and collagen I. Quantitative results are the means \pm standard error of 6 experiments, $* \mathrm{P}<0.05, * * \mathrm{P}<0.0$ l, between indicated groups. 

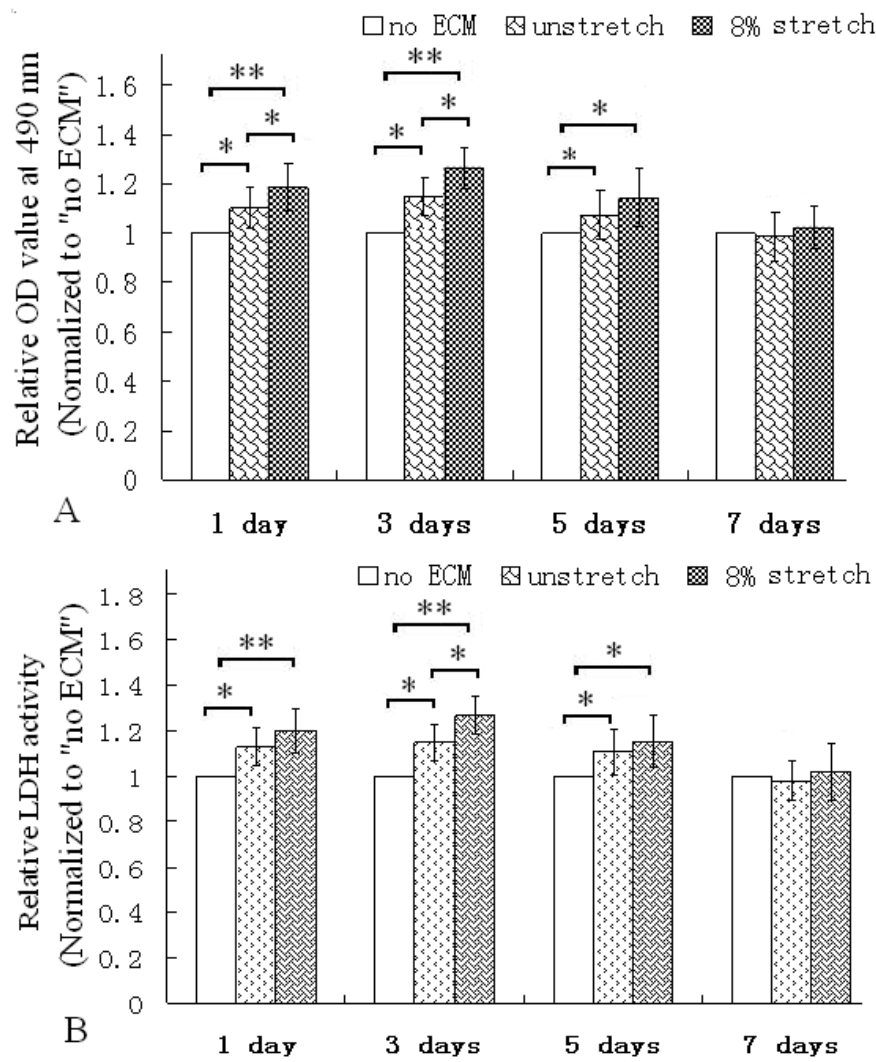

Fig 3. MTT assay and LDH activity assay of ventricular cells seeded on control (no ECM), ECM of unstretched cells (unstretch) coated on dishes, ECM of stretched cells (stretch of $8 \%$ for 7 days) on dishes. After culture for I, 3 and 5 days, ECM of stretched cells elevated the OD value of the cells (A); and improved LDH activity in cells (B). *P<0.05, ** $\mathrm{P}<0.01$, between indicated groups, $\mathrm{n}=6$.
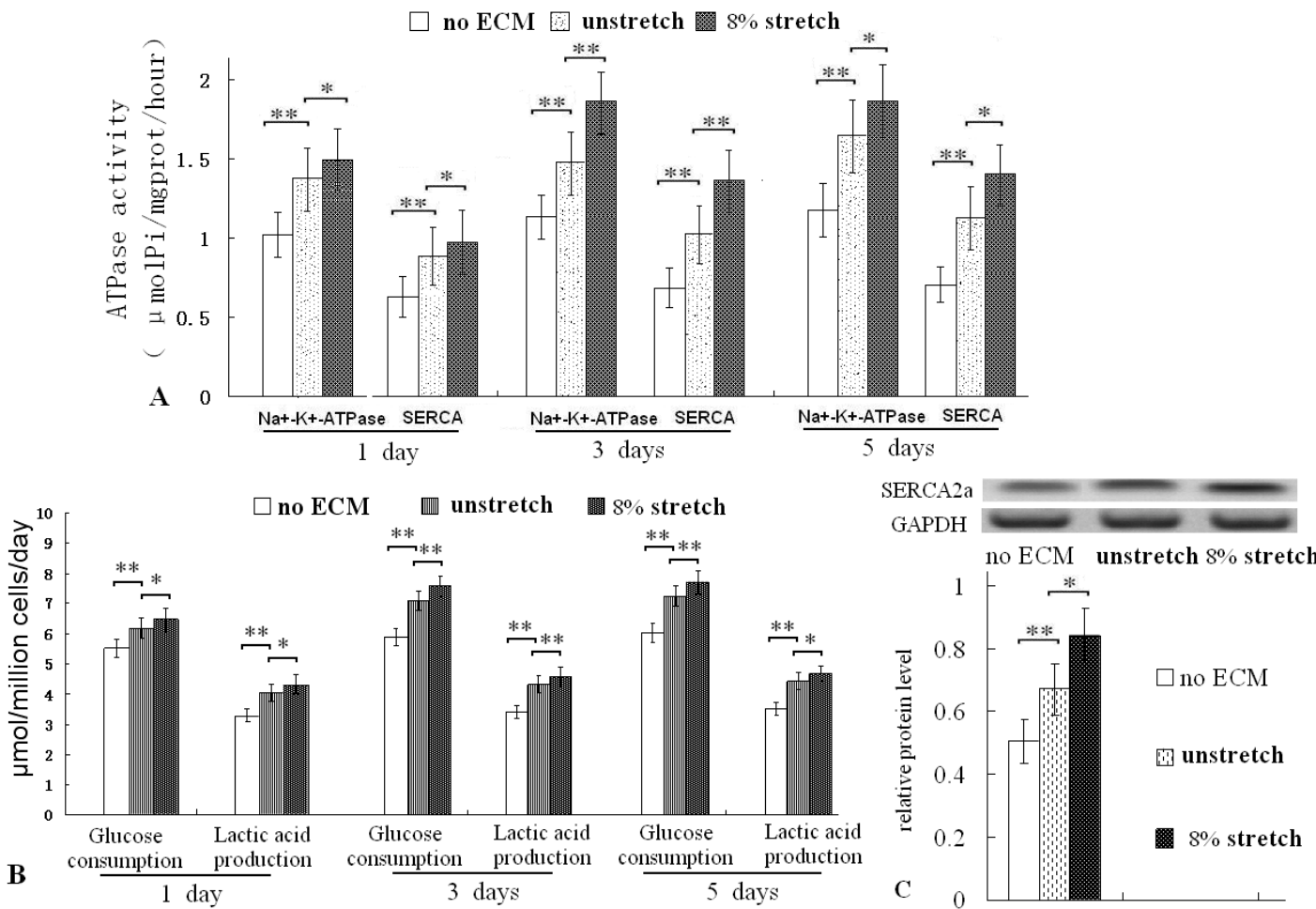

Fig 4. Assay of ATPase activity, glucose consumption and lactic acid production ratios of ventricular cells seeded on different ECMs, and Western blot of SERCA2a. After culture for I, 3 and 5 days, the ECM of stretched cells increased the activities of $\mathrm{Na}^{+} / \mathrm{K}^{+}$-ATPase and SERCA (A); and the ECM of stretched cells enhanced the glucose consumption and lactic acid production ratios of ventricular cells (B). After 3 days of culture, the mechanical stretch increased protein level of SERCA2a (C). $* \mathrm{P}<0.05, * * \mathrm{P}<0.0$ I, between indicated groups, $\mathrm{n}=7$. 

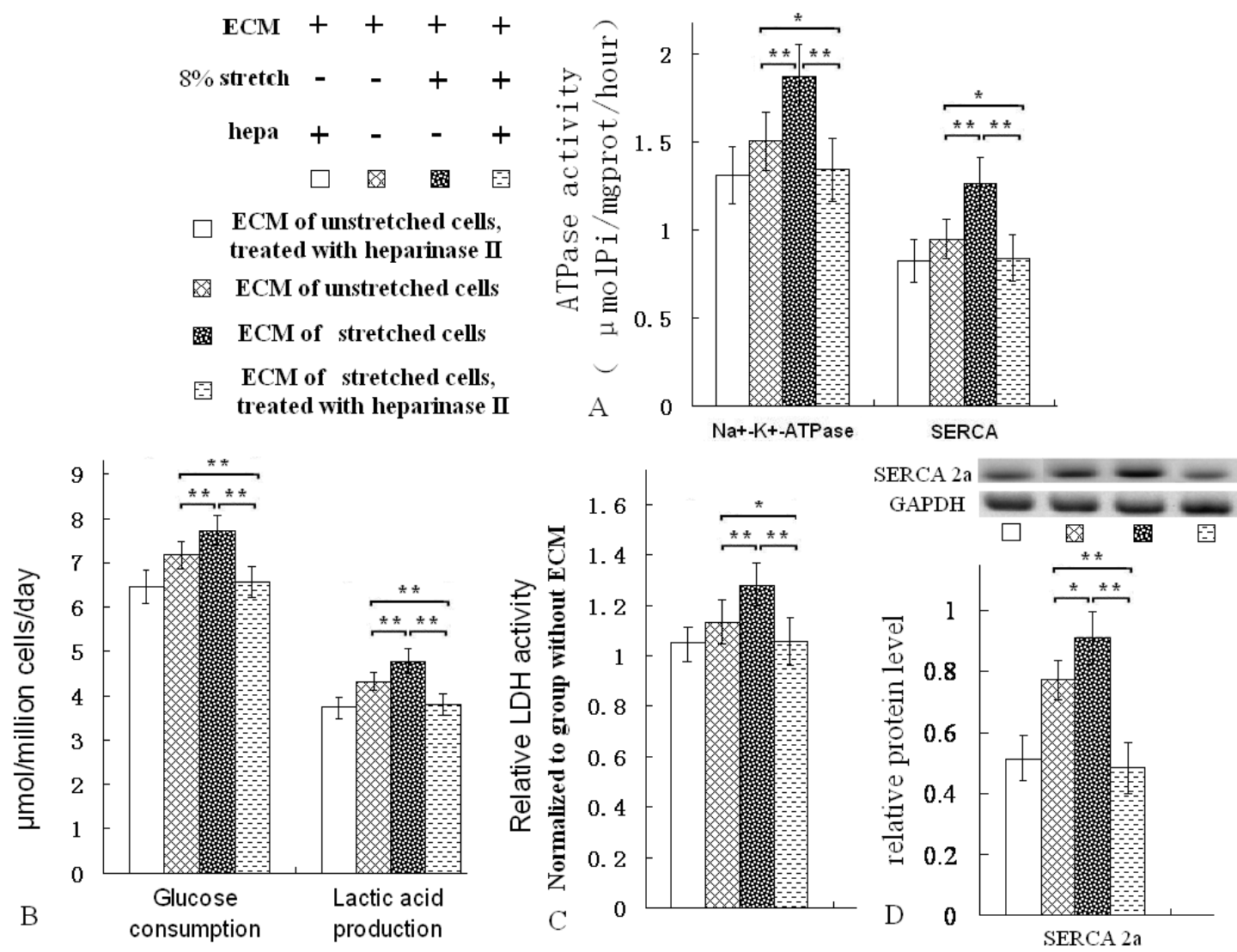

Fig 5. After the ECMs were treated with heparinase II [hepa, (Sigma, USA), $50 \mathrm{mU} / \mathrm{mL}, 37^{\circ} \mathrm{C}, 8 \mathrm{~h}$ ) and PBS washing, the ventricular cells were seeded on the ECM, then the metabolic activity of the cells was assayed after 3 days of growth. The treatment of hepa reduced activities of $\mathrm{Na}^{+} / \mathrm{K}^{+}-\mathrm{ATPase}$ and SERCA (A), decreased the glucose consumption and lactic acid production ratios (B), lowered LDH activity and SERCA2a protein levels (C, D). $*$ P $<0.05, * *$ $\mathrm{P}<0.0 \mathrm{I}$, between indicated groups, $\mathrm{n}=7$.

\section{Discussion}

ECM provides essential physical scaffold for cells, directs cells behavior including proliferation, growth, changes in shape, migration and differentiation (19). In the heart, the ECM network provides support for myocardial cell contraction and is responsible for the distribution of mechanical forces and signal transduction to individual cells via cell surface ECM receptor (20). So ECM of cardiac tissue is a very suitable scaffold material for cell/tissue engineering of cardiac tissue.

In this study, after removing of cells, we found that the ECM of mechanically stretched cardiac fibroblasts attached on menbranes, contained more collagen and GAG than those of unstretched cells. Collagen and GAG are the main components of ECM (1, $21)$, so we consider that the mechanical stretch $(4 \%$ or $8 \%, 1 \mathrm{~Hz}$ ) promoted formation of CF-ECM. Furthermore, Western blot result of fibronectin and collagen I confirmed that mechanical stretch promoted the ECM formation.

MTT assay was used to evaluate the viability and metabolic activity of the cultured cardiac cells (17).
$\mathrm{LDH}$ is an important oxidoreductase in cytoplasm; it plays an important role in the intracellular energy transport of cardiomyocytes (22). Assay of LDH activity is also used to evaluate the metabolic activity of cultured ventricular cells. In our study, the proliferation of cells in the cultures was inhibited with bromodeoxyuridine. CF-ECM of mechanically stretched cells enhanced viability and LDH activity of ventricular cells, which meant the CF-ECM of mechanically stretched cells, was more supportive for ventricular cells growth than that of unstretched cells.

$\mathrm{Na}^{+} / \mathrm{K}^{+}$-ATPase, is a ubiquitous transmembrane enzyme which transports $\mathrm{Na}^{+}$and $\mathrm{K}^{+}$across the plasma membrane (22-24). The a subunit of $\mathrm{Na}^{+} / \mathrm{K}^{+}$-ATPase couples the hydrolysis of ATP to the counter transport of $3 \mathrm{Na}^{+}$and $2 \mathrm{~K}^{+}$ions across the plasma membrane (25), the ATPase controls cell volume and regulates cardiac contraction (26), so the activity of the $\mathrm{Na}^{+} / \mathrm{K}^{+}$-ATPase is an important index of cardiac cell metabolism and function. SERCA, a $\mathrm{Ca}^{2+}$ ATP-dependent pump and key enzyme in the regulation of cytosolic $\mathrm{Ca}^{2+}$, critically determines the rate of cardiac muscle relaxation (27). SERCA is mainly represented by the SERCA2a isoform in the 
heart, it regulates the $\mathrm{Ca}^{2+}$ loading of the sarco(endo)plasmic reticulum and therefore the $\mathrm{Ca}^{2+}$ released and cardiac muscle contractility (27-28). Therefore, the activity of SERCA and the protein level of SERCA2a are determinant marks of cell function and metabolic activity, especially cardiac cell.

The results of the ATPase assays implied that CF-ECM of stretched cells elevated ventricular cells function and metabolism. The assays for glucose consumption and lactic acid production ratios indicated that the in vitro cultured ventricular cells consumed more glucose and produced more lactic acid. In this study, glucose was the main source of energy of ventricular cells cultured in vitro. Additionally, the CF-ECM of stretched cells elevated SERCA2a protein level. Therefore, the results further confirmed that the CF-ECM of stretched cells supported ventricular cell growth in vitro.

In this study, the in vitro cultured ventricular cells were composed of cardiomyocytes, fibroblasts, vascular endothelial cells, smooth muscle cells and other cell types $(15,29)$, which is consistent with normal cardiac tissue. Therefore, the evaluation of metabolism and function of cultured ventricular cells should correspond to that of intact cardiac tissue. These results indicated that CF-ECM of stretched cells supported ventricular cells' growth and promoted cellular metabolic activity in vitro, which meant the ECM of stretched cardiac fibroblasts was more supportive for in vitro culture of ventricular cells than that of unstretched cells. It is likely to contribute to ECM-modified biomaterial scaffold for cell/tissue engineering of heart.

ECM is a reservoir of growth factors and cytokines, such as bone morphogenetic protein, fibroblast growth factor, vascular endothelial growth factor, and these key molecules bind to either polysaccharide or protein constituents of ECM (30-32). These bioactive molecules binding to ECM regulate cell function and behavior, which suggests that ECMs produced in vitro display bioactivities, such as proliferative effect and inductive potential. In this study, mechanical stretch increased contents of collagen, fibronectin and GAG, so promoted ECM formation, this provided more space for bioactive proteins/peptides binding. Therefore, mechanical stretch influenced bioactivity of ECM.

GAGs are main components of ECM, they are linear heteropolysaccharides, characterized by different disaccharide unit repeats $(33,34)$. Heparin is one of the common structural elements of GAGs, heparinase II possesses the ability to cleave both heparin and heparan sulfate-like regions of GAG (35), it is a broad substrate-specificity GAG-lyase. In this study, treatment with heparinase II reduced GAG level greatly, which meant the GAG was decomposed or degraded and departed from the ECM. Furthermore, the treatment lowered metabolism-related indices (ATPase, glucose consumption ratio, lactic acid production ratio and SERCA2a protein level) of the ventricular cells seeded on the ECM, which indicated the GAG was involved in regulating metabolism of the cells cultured on the ECM.

More interestingly, in this study, after heparinase treatment, the effects of the two ECMs (produced by stretched cells and by unstretched cells) on metabolic activity or metabolism-related indices were nearly the same; the result indicated that the treatment of heparinase eliminated almost all of the GAG components which promoted metabolic activity. So it is worthwhile to investigate the influence of GAG on cells metabolic activity more detailed and deeply.

\section{Conclusion}

Mechanical stretch of $4 \%$ or $8 \%$ promotes ECM formation of cardiac fibroblasts in vitro, the ECM of mechanically stretched cardiac fibroblasts improves viability and metabolic activity of ventricular cells cultured in vitro. The GAG component of the ECMs is involved in regulating metabolic activity of ventricular cells. This was one of the first studies demonstrating that mechanical stretch promoted formation and improved bioactivity of cardiac fibroblasts' ECM produced in vitro.

\section{Acknowledgements}

This study was supported by National Natural Science Foundation of China (No. 11172062, No. 11302262, No. 11372351, No. 30970724, No. 10832012), Shandong Natural Science Foundation of PR China (ZR2010AL022), and this work was supported by funding from Shandong Provincial Key Laboratory of Functional Macromolecular Biophysics.

\section{Competing Interests}

The authors have declared that no competing interest exists.

\section{References}

1. Kleinman HK, Philp D, Hoffman MP. Role of the extracellular matrix in morphogenesis. Current Opinion in Biotechnology. 2003; 14: 526-532.

2. Adams JC, Watt FM. Regulation of development and differentiation by the extracellular matrix. Development. 1993; 117:118398.

3. Badylak SF. Regenerative medicine and developmental biology: the role of the extracellular matrix. Anat Rec B New Anat. 2005; 287: 3641.

4. Eghbali M, Czaja MJ, Zeydel M, Weiner FR, Zern MA, Seifter S, Blumenfeld OO. Collagen chain mRNAs in isolated heart cells from young and adult rat hearts. J Mol Cell Cardiol. 1988; 20(3): 267-276

5. Souders CA, Bowers SL, Baudino TA. Cardiac Fibroblast the Renaissance Cell Circ Res. 2009; 105(12):1164-1176

6. Carver W, Nagpal ML, Nachtigal M, Borg TK, Terracio L. Collagen expression in mechanically stimulated cardiac fibroblasts. Circ Res. 1991; 69(1):116-122.

7. Butt RP, Bishop JE. Mechanical load enhances the stimulatory effect of serum growth factors on cardiac fibroblast procollagen synthesis. J Mol Cell Cardiol. 1997; 29(4): 1141-1151. 
8. Husse B, Briest W, Homagk L, Isenberg G, Gekle M. Cyclical mechanical stretch modulates expression of collagen I and collagen III by PKC and tyrosine kinase in cardiac fibroblasts. Am J Physiol Regul Integr Comp Physiol. 2007; 293(5): R1898-R1907.

9. Baharvand H, Azarnia M, Parivar K, Ashtiani SK. The effect of extracellular matrix on embryonic stem cell-derived cardiomyocytes. J Mol Cell Cardiol. 2005; :38495-503.

10. Sreejit P, Verma RS. Cardiogel supports adhesion, proliferation and differentiation of stem cells with increased oxidative stress protection. Eur Cell Mater. 2011; 21:107-121.

11. Guo Y, Liu L, Hao QX et al. Effects of extracellular matrix produced in vitro on growth and differentiation of MC3T3-E1 cells. Chin J Biotech. 2011; 27:1606-12.

12. Qiang-cheng Zeng, Yong Guo, Lu Liu, Xi-zheng Zhang, Rui-xin Li, Chun-qiu Zhang, Qin-xin Hao, Cai-hong Shi, Ji-min Wu, Jing Guan. Cardiac fibroblast-derived extracellular matrix produced in vitro stimulates growth and metabolism of cultured ventricular cells. International Heart Journal. 2013; 54(1): 40-44

13. Lee AA, Delhaas T, Waldman LK, MacKenna DA, Villarreal FJ, McCulloch AD. An equibiaxial strain system for cultured cells. Am J Physiol. 1996; 27: C1400-C1408.

14. Yong Guo, Chun-qiu Zhang, Qiang-cheng Zeng, Rui-xin Li, Lu Liu, Qin-xin Hao, Cai-hong Shi,Xi-zheng Zhang. Mechanical strain promotes osteoblast ECM formation and improves its osteoinductive potential. BioMedical Engineering OnLine. 2012; 11:80.

15. Guo Y, Zhang XZ, Wei Y, Guo C, Li RX, Zeng QC, Zhang YJ. Culturing of ventricle cells at high density and construction of engineered cardiac cells sheet without scaffold. International Heart Journal. 2009; 50:653-662.

16. Berridge MV, Tan AS, Herst PM. Tetrazolium dyes as tools in cell biology: New insights into their cellular reduction. Biotechnology Annual Review. 2005; 11: 127-152.

17. Patra C, Talukdar S, Novoyatleva T et al. Silk protein fibroin from Antheraea mylitta for cardiac tissue engineering. Biomaterials. 2012; 33: 2673-2680.

18. Renner K, Amberger A, Konwalinka G, Kofler R, Gnaiger E. Changes of mitochondrial respiration, mitochondrial content and cell size after induction of apoptosis in leukemia cells. Biochim Biophys Acta. 2003; 1642: 115-123.

19. Daley WP, Peters SB, Larsen M. Extracellular matrix dynamics in development and regenerative medicine. J Cell Sci. 2008; 121:255-64.

20. Porter KE, Turner NA. Cardiac fibroblasts: at the heart of myocardial remodeling. Pharmacol Ther. 2009; 123:255-78.

21. Reilly GC, Engler AJ: Intrinsic extracellular matrix properties regulate stem cell differentiation. J Biomech. 2010; 43:55-62.

22. Saks, V A, Kupriyanov, V V and Elizarova, G V. Studies of energy transport in heart cells. J. Biol. Chem. 1980; 255: 755-763.

23. Skou JC, Esmann M. The Na+-K+-ATPase. J Bioenerg Biomembr. 1992; 24:249-261.

24. Lingrel JB, Kuntzweiler T. Na+-K(+)-ATPase. J Biol Chem. 1994; 269:19659-19662.

25. Blanco G, DeTomaso AW, Koster J, Xie ZJ, Mercer RW. The alpha-subunit of the Na, K-ATPase has catalytic activity independent of the beta-subunit. J Biol Chem. 1994; 269:23420-23425.

26. Shull GE, Greeb J, Lingrel JB. Molecular cloning of three distinct forms of the $\mathrm{Na}, \mathrm{K}$-ATPase alpha-subunit from rat brain. Biochemistry. 1986; 25: 8125-8132.

27. Periasamy M, Huke S. SERCA pump level is a critical determinant of Ca2+ homeostasis and cardiac contractility. J Mol Cell Cardiol. 2001; 33:1053-63.

28. Vangheluwe P, Raeymaekers L, Dode L, Wuytack F. Modulating sarco(endo)plasmic reticulum Ca2+ ATPase2(SERCA2) activity: cell biological implications. Cell Calcium. 2005; 38:291-302.

29. Zimmermann WH, Eschenhagen T. Cardiac tissue engineering for replacement therapy. Heart Fail Rev. 2003; 8: 259-269.

30. Pike DB, Cai S, Pomraning KR, Firpo MA, Fisher RJ, Shu XZ, Prestwich GD, Peattie RA. Heparin-regulated release of growth factors in vitro and angiogenic response in vivo to implanted hyaluronan hydrogels containing VEGF and bFGF. Biomaterials. 2006; 27: 5242-5251.

31. Faure C, Linossier MT, Malaval L, Lafage-Proust MH, Peyroche S, Vico L, Guignandon A. Mechanical signals modulated vascular endothelial growth factor-A (VEGF-A) alternative splicing in osteoblastic cells through actin polymerisation. Bone. 2008; 42: 1092-101.

32. Suzawa I, Takeuchi Y, Fukumoto S, Kato S, Ueno A, Miyazono K, Matsumoto T, Fujita T. Extracellular matrix-associated bone morphogenetic proteins are essential for differentiation of murine osteoblastic cells in vitro. Endocrinology. 1999; 140: 2125-2133.

33. Cechowska-Pasko M, Wolańska M, Pałłka J. Glycosaminoglycan-degrading enzymes in the skin of fasted rats. Comp Biochem Physiol B Biochem Mol Biol. 2002; 131: 551-557.

34. Zeng Y, Ebong EE, Fu BM, Tarbell JM. The structural stability of the endothelial glycocaly after enzymatic removal of glycosaminoglycans. PLoS ONE. 2012; 7: e43168.

35. Shriver Z, Hu Y, Sasisekharan R. Heparinase II from Flavobacterium heparinum. Role of histidine residues in enzymatic activity as probed by chemical modification and site-directed mutagenesis. J Biol Chem. 1998; 273(17):10160-7. 